\title{
Phylogenetic Targeting of Research Effort in Evolutionary Biology
}

\section{Citation}

Arnold, Christian and Charles L. Nunn. 2010. Phylogenetic targeting of research effort in evolutionary biology. American Naturalist 176(5): 601-612.

\section{Published Version}

doi:10.1086/656490

\section{Permanent link}

http://nrs.harvard.edu/urn-3:HUL.InstRepos:5342439

\section{Terms of Use}

This article was downloaded from Harvard University's DASH repository, and is made available under the terms and conditions applicable to Open Access Policy Articles, as set forth at http:// nrs.harvard.edu/urn-3:HUL.InstRepos:dash.current.terms-of-use\#OAP

\section{Share Your Story}

The Harvard community has made this article openly available.

Please share how this access benefits you. Submit a story.

Accessibility 
${ }^{1}$ Department of Human Evolutionary Biology, Harvard University, 11 Divinity Avenue,

21 Keywords: comparative method, phylogeny, correlated evolution, taxon sampling, pairwise 22 comparison 
27 Many questions in comparative biology require that new data be collected, either to build 28 a comparative database for the first time or to augment existing data. Given resource 29 limitations in collecting data, which species should be studied to increase the size of 30 comparative datasets? By taking the hypotheses, existing data relevant to the hypotheses, and 31 a phylogeny, we show that a method of "phylogenetic targeting" can systematically guide data 32 collection while taking potentially confounding variables and competing hypotheses into 33 account. Phylogenetic targeting selects potential candidates for future data collection using a 34 flexible scoring system based on differences in pairwise comparisons. We used simulations to 35 assess the performance of phylogenetic targeting, as compared to a less systematic approach 36 of randomly selecting species (as might occur when data have been collected without regard 37 to phylogeny and variation in the traits of interest). The simulations revealed that phylogenetic 38 targeting increased the statistical power to detect correlations and that power increased with 39 the number of species in the tree, even when the number of species studied was held constant. 40 We also developed a web-based computer program called PhyloTargeting to implement the 41 approach (http://phylotargeting.fas.harvard.edu). 
44 The comparative method has played a major role in uncovering adaptive trait evolution in

45 biological systems (Harvey and Pagel 1991; Martins 2000; Pagel 1999; Ridley 1983). The

46 comparative method has revealed, for example, links between mating systems and sperm

47 competition in primates (Harcourt et al. 1981) and other animals (Hosken 1997; Moller 1991).

48 The comparative method also supported a model of sexual selection in which females choose

49 males based on their ability to resist parasites (Hamilton and Zuk 1982), and it has been used

50 to probe the origins of both parasitic and symbiotic associations (e.g., Hugot 1999; Lutzoni et

51 al. 2001). More recently, comparative methods have been applied to study phylogenetic

52 community ecology (Webb et al. 2002), for example in the context of the phylogenetic over-

53 dispersion of mammalian communities (Cooper et al. 2008). The comparative method also

54 can be used to address conservation issues (Fisher and Owens 2004), such as questions

55 involving the factors that influence rates of extinction (Purvis et al. 2000b) and how the

56 phylogenetic clumping of conservation threat status can lead to greater loss of phylogenetic

57 diversity when species go extinct (Purvis et al. 2000a).

58 A comparative analysis requires data on a set of species relevant to a hypothesis of

59 interest. Usually, however, data are available for only a fraction of the species in a clade, and

60 data collection in both the field and laboratory is expensive and time-consuming. A proper

61 selection of species to study is a non-trivial and multi-faceted problem (Garland 2001;

62 Westoby 2002) that has rarely been addressed in a systematic way. Instead, species are often

63 chosen either randomly or subjectively (Faustino 2008; Westoby 1999) because they are of

64 "particular (and perhaps irrational) interest" (Garland 2001, p.119). Two problems are

65 introduced when species are chosen in an unsystematic way. First, the full range of variation

66 is not used to test the hypotheses. Second, taxonomic gap bias may occur, meaning that data

67 collection has been focused on a few "popular" lineages. These different kinds of biases -

68 incomplete variation and gap biases - can make a momentous difference to the conclusions 
69 one draws. In studies of primates, for example, results of comparative research are likely to

70 change when the sample is tilted towards terrestrial species, rather than those that live in the

71 trees, because terrestrial species possess larger body masses, exhibit different locomotor

72 patterns, and live in larger social groups (Clutton-Brock and Harvey 1977; Martin 1990; Nunn

73 and van Schaik 2002).

74 To address these issues, methods are needed to quantify potential biases in comparative

75 datasets and to identify the species that should be studied in the future. Indeed, it is common

76 to read in write-ups of comparative research that further sampling is needed to validate the

77 findings, either because the sample sizes were small or the sample was biased towards

78 particular species within a clade (e.g., in the study of sleep patterns: Capellini et al. 2009;

79 Nunn et al. 2009; Roth et al. 2006). Unfortunately often, however, only general guidelines for

80 this selection process have been given, and these guidelines are often specific to the question

81 of interest (Westoby 2002). To our knowledge, no method yet exists that is flexible and

82 specific enough to address the crucial task of prioritizing future research in light of specific

83 hypotheses about the apportionment of variation in relation to one or more ecological factors.

84 Only a handful of studies have investigated ways of systematically identifying species to

85 study. For example, Ackerly (2000) compared the performance of different taxon sampling

86 strategies and found that their statistical performance differed substantially. One of the

87 algorithms he examined is based on the pairwise comparison approach (Felsenstein 1985,

88 p.13; Maddison 2000; Møller and Birkhead 1992; Oakes 1992; Purvis and Bromham 1997;

89 Read and Nee 1995) and identifies meaningful comparisons by selecting species pairs that

90 differ by a certain amount in the independent variable, following the suggestion of Westoby

91 (1999). Although it overestimates the magnitude of the correlation, Ackerly (2000) showed

92 that this design increases the statistical power to detect correlated evolution (see also Garland

932001 and Garland et al. 1993). One major weakness of the method is that the threshold for

94 when differences are "large" is arbitrary, dependent on the dataset, and must be set manually, 
95 which limits its applicability considerably. Mitani et al. (1996) considered sampling strategies

96 in relation to testing competing hypotheses, while Read and Nee (1995) discussed the need to

97 identify pairs that contribute for or against hypotheses. Similarly, Maddison (2000) presented

98 a methodology for choosing species pairs in which each pair is "a comparison relevant for the

99 question of interest" (p. 198). However, his method is designed for binary rather than

100 continuously varying data, and it can only handle fully bifurcating trees and thus does not

101 provide enough flexibility for identifying meaningful comparisons with real data.

102 The method of pairwise comparisons has been used frequently to identify meaningful

103 comparisons. Several reasons exist for using pairwise comparisons. For example, the method

104 of pairwise comparison relies on fewer assumptions (Ackerly 2000; Hearn and Huber 2006;

105 Maddison 2000) than other methods. Thus, unlike phylogenetically independent contrasts

106 (PIC) (Felsenstein 1985; Garland et al. 1992; Harvey and Pagel 1991), pairwise comparison

107 does not require a specific model of evolution or the estimation of states at interior nodes. In

108 addition, some sets of species within a larger clade might not be directly comparable in

109 standard implementations of comparative methods, such as PIC. In mammalian sleep, for

110 example, some cetaceans sleep with only one half of their brains (Lyamin et al. 2008), making

111 it difficult to compare the measurements of sleep in cetaceans to other mammals. The method

112 of selecting specific pairwise comparisons provides a way to limit comparisons so that

113 cetaceans are compared only to other cetaceans, and non-cetaceans are compared only to non-

114 cetaceans. Similarly, some behavioral experiments might require similar sensory modalities or

115 cognitive ability among species in the dataset. Pairwise comparisons of some close relatives

116 may be more appropriate for selecting species for focused comparative experiments that take

117 these factors into account.

118 When using the method of pairwise comparisons, it is important that all pairs are

119 phylogenetically independent, i.e. no branches are shared among the comparisons (Felsenstein

120 1985; Maddison 2000). In Figure 2, for example, different sets of phylogenetically 
121 independent pairs (which we call a "pairing," see Maddison 2000) are shown for each tree.

122 Thus, when selecting phylogenetically independent pairs, the selection of a particular pair

123 constrains which other pairs can be selected.

124 Here, we present a new approach, which we call "phylogenetic targeting," to 125 systematically identify the species to study in the future. Phylogenetic targeting is a taxon 126 sampling approach that aims to prioritize future research by identifying species that should be 127 studied in a target-oriented way under consideration of the specific hypotheses and data. It is 128 not a new way to analyze comparative data or a substitute for existing analysis methods, but 129 rather draws on existing methods in comparative biology. This method uses the pairwise 130 comparisons approach and is based on a scoring system that incorporates phylogeny and data 131 on variables relevant to testing hypotheses, specifically involving the predictor and response 132 variables in a comparative test. The predictor variables can include potentially confounding 133 variables or variables relevant to testing alternative hypotheses for an association. If external 134 information suggests that comparisons should be restricted taxonomically or in relation to 135 existing data, one can use the method to limit which species to compare.

136 After assigning a score for each pair of species, phylogenetic targeting uses a newly 137 developed algorithm to select the set of phylogenetically independent pairs of species that 138 offer greater statistical power to test the hypothesis once data have been collected on the 139 dependent variable. After collecting data, pairwise contrasts for the targeted species pairs can 140 be used to test hypotheses, or one can use standard comparative techniques for testing 141 correlated character evolution (Figure 1). This decision is up to the investigator and depends 142 on the actual hypotheses, data and analysis preferences (see Discussion). We use computer 143 simulations to assess the degree to which phylogenetic targeting increases statistical power for 144 detecting correlated trait evolution, as compared to random sampling of species. We also 145 implemented the method online (http://phylotargeting.fas.harvard.edu). We anticipate that the 146 general approach developed here for pairwise comparisons can be developed for use with 
147 additional comparative methods, such as PIC or generalized least squares approaches, and we

148 discuss some of these potential extensions.

\section{METHODS}

151 The method requires a phylogeny and one or more explicit hypotheses that offer predictions

152 for how variation in one trait $\left(X_{1}\right)$ correlates with variation in another trait that is common to

153 all the hypotheses and, because it is not known in all the species, is the "target" of the analysis

$154\left(Y_{t}\right)$ (Figure 1). We call this association between $Y_{t}$ and $X_{l}$ the primary hypothesis. Additional

155 hypotheses, if desired, are implemented through traits $X_{2} \ldots X_{n}$, which relate to competing

156 hypotheses or potentially confounding variables. The goal of the method is to identify species

157 that should be studied with regard to $Y_{t}$ by using phylogenetic relationships and data already

158 collected for the $X$ traits. Thus, a species cannot be included in a phylogenetic targeting

159 analysis if data on $\mathrm{X}$ are lacking for that species. We assume that larger evolutionary changes

160 in $\mathrm{X}_{1}$ provide higher statistical power for comparative tests to test the hypotheses, because it

161 increases the available range of variation (Garland 2001; Garland et al. 2005; Westoby 1999;

162 Westoby et al. 1998). We also assume that the characters show a linear relationship. Different

163 targeting analyses are likely to focus on a primary hypothesis and various combinations of 164 alternative hypotheses, and both discrete and continuous traits can be used. Scores are 165 calculated so that higher values indicate more preferred species to study, based on user166 defined criteria involving control of confounding variables, testing of alternative hypotheses, 167 and availability of data on $Y_{t}$ for one or more species in a clade.

\section{Calculating pairwise comparisons}

170 The analysis starts by calculating all possible $n *(n-1) / 2$ pairwise comparisons. In the tree

171 shown in Figure 2, for example, 15 comparisons can be constructed. The method thus does

172 not rely on using only pairs of sister species, as pairs of more distantly related species could 
173 also offer compelling tests of the hypotheses (Maddison 2000; Read and Nee 1995; Westoby

174 1999). Pairwise comparisons with missing data in any of the traits except $Y_{t}$ are excluded. In

175 addition, certain species can be excluded manually from the analysis, for example in cases

176 where an experiment can be applied to only certain species on the tree.

177 If discrete characters with more than two possible states are used, they can be treated

178 as ordered (costs between different pairs of states are different, as a particular sequence exists

179 in which the states must occur through evolution) or unordered (every state change is equal, as

180 each state can directly be transformed into any other state) (Slowinski 1993).

\section{Calculating scores for models with a single predictor $\left(Y_{t}\right.$ and $\left.X_{I}\right)$}

183 For predictions that only involve a primary hypothesis (i.e., only one independent variable), 184 phylogenetic targeting uses a scoring system that maximizes the variability in $X_{1}$. In other 185 words, species pairs are targeted that differ the most in $X_{1}$. If we were interested in hypotheses 186 that involve body mass as an independent variable, for example, phylogenetic targeting gives 187 pairs with the largest differences in body mass higher scores. Thus, pairwise comparisons 188 with big differences in $X_{I}$ are scored more positively, whereas smaller differences are scored 189 less positively. These contrasts are then standardized to the scale 0 to 1 , with a difference of 0 190 assigned a score of 0 and the largest difference in all considered pairs assigned a score of 1. 191 Note that even if no zero contrasts are found in the data, the method fixes this as the lowest 192 contrast. All other differences are assigned a score between 0 and 1 by applying a linear 193 scaling transformation. We call this the score of $X_{1}$.

194 If $X_{1}$ is an unordered discrete character, the score will be either 0 or 1 regardless of the 195 actual difference in character state assignments, whereas the difference is scored on an 196 interval between 0 and 1 in the case of an ordered character, with the maximum number of 197 character steps scored as 1. 
200 Models that incorporate additional traits enable the testing of different kinds of hypotheses 201 (e.g., mutually exclusive and non-mutually exclusive), and they can be used to control for 202 confounding variables. For each $X_{2} \ldots X_{n}$, a separate scoring mechanism is defined in which 203 larger contrasts have either a negative or a positive influence on the overall score. The 204 decision for whether larger differences in each of the $X_{2}$ to $X_{n}$ variables is scored higher or 205 lower depends on whether the variables reflect confounding variables or a desire to 206 distinguish among competing hypotheses. To simplify discussion in what follows, we 207 consider a case in which only one additional variable is included; thus $Y_{t}=\mathrm{f}\left(X_{1}, X_{2}\right)$. Further 208 details on the specifics of scoring are given below.

209 To control for confounding variables, the goal is to minimize variation in the predictor

210 variable that corresponds to the confounding variable of interest, i.e. $X_{2}$. Thus, pairwise 211 comparisons in $X_{2}$ that make the absolute value of change in a particular confounding variable 212 as small as possible are scored higher, whereas pairwise comparisons with bigger differences 213 are scored lower ( Score $_{\mathrm{NC}}$, i.e. the score from standardizing the covariate for "no change"). 214 The smallest pairwise contrast is assigned a score of 1, whereas the maximum pairwise 215 contrast is assigned a score of 0 . All other differences are assigned a score between 0 and 1.

216 To address mutually exclusive hypotheses, the goal is to maximize scores for $X_{2}$ that

217 differ maximally from contrasts in $X_{1}$. Two different scoring options can be applied that both 218 target big differences, but differ in how they score these differences. The first option scores 219 differences in $X_{2}$ in the opposite direction as the difference in $X_{1}$ positively and differences in 220 the same direction as $X_{I}$ negatively (Score OD , i.e. the score from standardizing covariate in the 221 "opposite direction"). The biggest difference in the opposite direction is assigned a score of 1, 222 whereas the biggest difference in the same direction is assigned a score of -1. A difference of 2230 is assigned a score of 0 . The smallest pairwise contrast is always assigned 0 even if no 224 pairwise comparison has a difference of 0 in this trait, as this ensures that all non-zero 
225 differences are assigned a score different from 0 . All other differences are assigned a score

226 between -1 and 1 by applying a linear scaling transformation, which is calculated separately

227 for positive and negative contrasts. The second option is the opposite of the first option; that

228 is, differences in the opposite direction from the difference in $X_{1}$ are scored negatively and 229 differences in the same direction are scored positively (Score $\mathrm{SD}$, i.e. the score from 230 standardizing covariate in the "same direction"). For example, this option might be useful if 231 an increase in $X_{1}$ is predicted to reduce $Y_{t}$ while an increase in $X_{2}$ is predicted to increase $Y_{t}$. 232 Thus, it is necessary to give higher scores to contrasts in the same direction for $X_{1}$ and $X_{2}$ to 233 distinguish among the hypotheses.

234 For models with covariates, the direction of change for $X_{2} \ldots X_{n}$ always refers to the 235 direction of change in $X_{1}$, e.g. a positive value means that the direction of change is the same 236 as in $X_{1}$. By doing so, we force the difference in $X_{1}\left(\Delta_{\text {raw }}\right.$, see Table 1$)$ to be positive and 237 achieve consistency with other widely-used programs, such as CAIC (Purvis and Rambaut 238 1995) and PDAP-Mesquite (Midford et al. 2005). This "positivization assumption" also helps 239 to make sense of the other trait differences and their directions when using the computer 240 program, as it becomes possible to determine whether other pairwise comparisons are 241 consistently positively or negatively associated with $X_{1}$ (e.g., if $X_{2}$ is positive, it must be in the 242 same direction as $X_{1}$ ). Although not strictly necessary for the algorithms implemented here, 243 this helps guide manual selection of contrasts in the web-based implementation of 244 phylogenetic targeting.

\section{Summed score and standardizing scores for branch lengths}

For each pairwise comparison, the scores for all traits are summed up to define the

248 summed score (see Table 1 for a case involving $X_{2}$ as a confounding variable, i.e. Score $\mathrm{NC}_{\mathrm{NC}}$.

249 The summed score combines the information from all traits and thus represents the strength of 
250 a pair for testing the hypotheses. For models with only $Y_{t}$ and $X_{1}$, the summed score thus 251 equals the score of $X_{1}$.

Regardless of the scoring model, the summed score can sometimes be uninformative

253 when compared among different pairs because the more divergent two species are, the more

254 likely it is that they evolved bigger differences. In other words, different pairs will have 255 different expected amounts of change (i.e., variance). In our approach, we overcome this 256 problem by normalizing the summed score by its expected variance (square root of the sum of 257 the branch lengths that connect the two species) (Felsenstein 1985; Garland et al. 1992). We 258 call this the standardized summed score. By doing so, all pairwise comparisons have a 259 common variance as required by most statistical tests (see also Discussion).

260 Table 1 summarizes and applies the scoring system to the dataset in Figure 2, based on 261 controlling for $X_{2}$ as a confounding variable ( Score $_{\mathrm{NC}}$ ). Different standardized summed scores 262 would be obtained if we treated $X_{2}$ as representing a competing hypothesis, and depending on 263 the expected direction of $X_{2}$ in the context of competing hypotheses (see columns for Score $\mathrm{SD}$ 264 and Score $\mathrm{OD}$ in Table 1).

\section{Availability variable}

267 In addition to manually excluding species from an analysis, it is possible to define an "availability variable" to automatically exclude species or pairs in relation to the availability

269 of data for $Y_{t}$. One can thus use the availability variable to identify other species that should be 270 studied in the context of existing data on $Y_{t}$. An availability variable also provides a way to 271 quickly "pinpoint" where the missing data points are in a phylogenetic context, which can 272 help to identify biases in the distribution of the studied species.

273 The availability variable must be a discrete binary variable that identifies whether or 274 not data are available for $Y_{t}$ for a particular species. For example, consider the scenario in 275 Figure 2, in which $B_{t}$ is the availability variable. Possible options would be to only consider 
276 pairs where data are available for both species that form the pair (exclusion of all pairs except 277 s1-s5), for one species (exclusion of pairs s1-s5 and all combinations of s2, s3, s4 and s6), for 278 at least one species (as before, but not s1-s5) and for none of the species (exclusion of the nine

279 pairs with s1 and s5). This scoring procedure thus can be used in a variety of ways. For 280 example, if the availability variable indicates that data are available for only a fraction of the 281 species, the majority of the pairs will be excluded if the option is chosen to consider only pairs 282 where one species has already been studied and data are needed for the other species. In such 283 a case, only those pairs containing one studied species and one that has yet to be studied 284 remain. It can thus be seen as an additional selection factor that effectively constrains the 285 species that will be targeted.

\section{Maximal pairing algorithm}

The actual selection of species is performed by a dynamic programming algorithm that

289 we call maximal pairing. The maximal pairing algorithm is a general optimization algorithm 290 and selects pairs of species that are phylogenetically independent. In contrast to PIC, where 291 pairs can also involve internal nodes on the tree, the maximal pairing algorithm selects only 292 pairs between the tips of the tree. The selection of pairs is based on the summed score for each 293 pair, and the algorithm determines the set of phylogenetically independent pairs that 294 maximizes the sum of the individual summed scores (Table 1). This criterion is thus assumed 295 to maximize the power to test the hypotheses given constraints on maintaining phylogenetic 296 independence. With large datasets, it is difficult to find the maximal pairing manually, due to 297 the large number of possible pairings and the complex phylogenetic dependence of pairs that 298 must not share a branch (Figure 2). Despite some differences that involve execution time and 299 representation of polytomies, the maximal pairing algorithm also works for polytomous trees 300 (see Online Appendix A for more details). 
For models that involve only $X_{1}$, for example, the maximal pairing generally selects

302 pairs of closely related species that maximize differences in $X_{1}$, and those pairs are often

303 distantly related to the other pairs that are selected. In a comparative test, such a design is

304 considered to be especially powerful (Garland et al. 2005). If, however, an additional trait $X_{2}$

305 is used to control for confounding variables (thus scoring small differences in $X_{2}$ higher using

306 Score $_{\mathrm{NC}}$ ), the algorithm both maximizes differences in $X_{1}$ and minimizes differences in $X_{2}$.

307 Conversely, if one aims to maximize differences in $X_{2}$ (thus scoring larger differences in $X_{2}$ 308 opposite to $X_{1}$ higher with Score $\left.{ }_{\mathrm{OD}}\right)$, the algorithm maximizes differences in $X_{1}$ and 309 maximizes differences in $X_{2}$ opposite in sign to $X_{1}$. Similar logic applies to Score ${ }_{\mathrm{SD}}$. It is 310 worth noting, however, that due to the phylogenetic constraints and the standardizing of 311 contrasts, the maximal pairing does not simply select the pairs with the most extreme 312 character differences; instead, pairs with small differences among closely related species are 313 also frequently selected.

\section{Simulations}

We compared the performance of phylogenetic targeting to random selection of species

317 using simulations. The aim of the simulations was to generate data with known degrees of 318 correlation between pairs of variables, and then to select subsets of species either randomly or 319 using phylogenetic targeting. To perform the simulations, we first generated phylogenetic 320 trees and character data using the GEIGER package (Harmon et al. 2008) in $R$ (R 321 Development Core Team 2009) according to a uniform birth-death process $(b=0.15, d=0)$. We 322 created 1500 random phylogenies for a series of $N=50,70$, and 90 taxa. We then simulated 323 character evolution for two continuously varying characters on each tree using five different 324 models of evolution (Table 2) with character states $(0,0)$ at the root of the tree. When 325 simulating the non-Brownian motion models of evolution, we first transformed the tree in 326 Geiger (Harmon et al. 2008), simulated traits on the transformed tree, and then analyzed the 
327 data on the original tree, thus simulating a case where the branch lengths failed to accurately

328 reflect trait evolution (see Online Appendix B). Characters were simulated with a variance of

329 one and correlations of 0 and 0.5 , respectively. This yielded 4500 datasets with varying

330 numbers of species and known evolutionary correlations among the characters.

331 Using these data and phylogenies, we then selected subsets of species randomly and

332 using phylogenetic targeting. In each simulation file, we selected the first simulated trait as

$333 X_{1}$; the second variable was assumed to be $Y_{t,}$. We also standardized the scores. The maximal

334 pairing was then calculated, and we selected the six highest scoring pairs. We also randomly

335 selected six phylogenetically independent pairs. To investigate whether the number of

336 selected pairs impacts statistical performance, all analyses were repeated using 9 pairs and 12

337 pairs.

338 To evaluate statistical properties of both sampling approaches, we performed standard 339 statistical tests based on the selected pairwise comparisons. For that, we used the character

340 differences for $\mathrm{X}_{1}$ and $\mathrm{Y}_{\mathrm{t}}$ for the selected pairs and standardized them by their expected

341 variance (square root of the sum of the branch lengths that connect the two species). We

342 tested for a significant correlation between both characters using the correlation coefficient

343 through the origin (Garland et al. 1992), with significance based on $\alpha=0.05$ using a t-test

344 with $N-2$ degrees of freedom. We determined Type I error rates (incorrectly rejecting a true

345 null hypothesis of no association between traits) and statistical power (probability of rejecting

346 a false null hypothesis) for both sampling approaches. Type I error rates were calculated as

347 the proportion of significant results based on $\mathrm{p}=0.05$ for datasets in which $\mathrm{r}=0$, while

348 statistical power was based on the proportion of significant results for datasets in which $\mathrm{r}=0.5$.

349 In addition to tests based on pairwise comparisons, we performed tests based on the full

350 set of independent contrasts. We did this because many users may be interested in using a full

351 set of contrasts, yet the method operates by examining pairwise comparisons. Thus,

352 understanding the statistical performance of phylogenetic targeting when used with PIC is an 
important step and expands its application spectrum. After pruning the tree to the subset of

354 selected pairs, we calculated PIC (Felsenstein 1985) using the APE package (Paradis et al.

355 2004). We tested for a significant correlation between both characters using the methods

356 described in the previous paragraph.

357 We also tested how the inclusion of randomly selected, non-targeted species affects the results. This simulates a common situation because data are often already available for some species but missing for others. Specifically, we examined how including $k$ random species 360 affects the results for tests based on pairwise comparisons and PIC (with $k$ ranging from 2 to 10 in steps of 2). We included these additional species from the remaining set of species that were not selected by phylogenetic targeting (and thus without using the availability variable). Lastly, we analyzed how much of the original range of variation in the simulated data was available after pruning the data to the selected species. This gives insights to the range of variation that is available for hypothesis testing under the two sampling techniques.

\section{RESULTS}

\section{PhyloTargeting program}

We created a freely available computer program - PhyloTargeting - that implements the 370 phylogenetic targeting approach. It is web-based, takes the data as a Nexus file (Maddison et al. 1997) and provides a user-friendly, interactive, step-by-step interface, a variety of analysis

372 options, and graphical visualizations of the results. The program is publicly available at 373 http://phylotargeting.fas.harvard.edu.

\section{Simulations}

The simulations revealed that phylogenetic targeting substantially increases the range of

377 biological variation that is sampled relative to random sampling (Figure 4). Phylogenetic 378 targeting also provided substantially higher statistical power for detecting a true relationship 
379 (Figure 5). This held for both the pairwise tests and tests based on PIC. For the pairwise tests, 380 Type 1 error rates for $\alpha=0.05$ were elevated if the number of selected pairs was small, but 381 decreased to the expected level when more pairs were selected. For the tests based on PIC, 382 Type I error rates were close to the expected level in all scenarios. Importantly, Type 1 error 383 rates under random sampling and phylogenetic targeting were generally indistinguishable.

384 More details are provided in Online Appendix C.

385 Increasing the number of pairs that are selected by the sampling algorithms increased 386 statistical power, as expected (Figure 5). For the pairwise tests, it also decreased Type 1 error 387 rates. The number of taxa per tree, however, revealed a more surprising effect. Even when 388 holding the number of pairs constant, the statistical power increased with the number of taxa 389 in the clade under phylogenetic targeting, and Type 1 error rates did not increase (Figure 5). If 390 species are selected randomly, however, power did not increase with increasing clade size.

391 When the true correlation was 0.5 , mean values of $r$ were elevated, and moreover 392 increased with the number of species per tree (see Online Appendix C). Thus, a sampling 393 regime based on phylogenetic targeting resulted in biased estimates of evolutionary trait 394 correlations when $r \neq 0$, whereas a random selection of species resulted in no bias. Importantly, 395 however, no bias was found when the true correlation was 0 , as shown in the results for Type 396 I error rates. Furthermore, the bias decreased substantially if additional, randomly selected 397 species were included (see Discussion and Online Appendix C).

398 The results highlighted above are for a Brownian motion process of character evolution. 399 For the alternative models that we tested (see Online Appendix B), results were comparable. 400 However, for most of these analyses, Type 1 error rates were highly elevated and statistical 401 power was reduced under the two sampling approaches and for PIC on the full tree (which we 402 used as a control). Not surprisingly, the pairwise tests showed substantially less elevated Type 4031 error rates if model assumptions were violated, possibly because the method of pairwise 404 comparisons relies on fewer assumptions. 


\section{DISCUSSION}

407 Comparative studies generally make use of available data. Here we show that the 408 comparative approach can also be used to target species for future data collection. By

409 applying the phylogenetic targeting concept, we can identify species that offer higher power 410 to test predictions of a comparative hypothesis. Moreover, phylogenetic targeting provides a 411 way to control for confounding variables when selecting species for further study, or to test 412 competing hypotheses. The method will most likely be used to augment existing data, but it

413 can also be applied to generate new datasets in the context of finite resources for data 414 collection.

415 A major strength of the approach is that phylogenetic information is incorporated when 416 selecting species to study (Garland 2001; Garland et al. 2005), thus ensuring that the selected 417 pairs are phylogenetically independent of one another. This makes it possible to analyze the 418 data using standard statistical methods (i.e., pairwise tests). However, the simulations revealed 419 that compared to PIC, statistical power is reduced (see also Ackerly 2000). This may be due 420 to the fact that for pairwise differences, the number of data points is reduced by a factor of 421 approximately 2 , because only the tips of the tree are contrasted and not the interior nodes of 422 the tree. Furthermore, the bias in estimating the correlation coefficient is increased with 423 pairwise comparisons. We thus advise users to analyze the selected species with standard 424 comparative methods based on the full set of contrasts whenever possible instead of using the 425 differences for the selected pairs directly.

426 The simulation results revealed that phylogenetic targeting provides many advantages 427 compared to a random selection of species for detecting correlated trait evolution. Statistical 428 power was strongly increased in all cases that we examined. Phylogenetic targeting used a 429 higher percentage of the available range of variation for a character, as compared to random 430 sampling of species. Thus, we can be more certain that the pattern holds generally across the 
431 clade of organisms rather than, for example, only among the species that are larger in body

432 size or more amenable to study. Surprisingly, the simulations also revealed that statistical

433 power increased with the number of species per tree, even when the number of taxa selected

434 for study remained constant. Type 1 errors, however, were always close to the nominal level

435 and undistinguishable between phylogenetic targeting and random species sampling. Thus,

436 applying the method to larger clades resulted in increased power without increasing the

437 number of pairs examined, probably because having more taxa increased the magnitude of the

438 differences that can be selected overall (which increased the ability to detect a correlation).

439 Phylogenetic targeting should be used with caution when one wants to determine the 440 magnitude of a correlation. Similar to the pairwise approach of Westoby (1999), it 441 overestimates the correlation coefficient (Ackerly 2000). This was true for both the pairwise 442 tests and PIC, and the bias was stronger with the pairwise tests. The simulations also revealed 443 that this overestimation increases with the number of species per tree, thus mirroring the 444 increase in power. In the context of applying the method to real-world data in which data for $445 Y_{t}$ are already available for some of the species, however, simulations confirmed that this bias 446 decreases substantially with the number of randomly selected species for which data are 447 already available. For most questions of interest that we envision, data are often available on $448 Y_{t}$ for a number of species, often comprising a majority of the species in the dataset. When 449 such data are available, inclusion of already available data in subsequent analysis after 450 applying phylogenetic targeting is highly recommended. Alternatively, users can implement 451 the availability variable option described above to more fully integrate decisions about future 452 data collection with already studied species. Furthermore, as noted above, the bias is likely to 453 decrease if additional traits representing confounding variables or alternative hypotheses are 454 included in the analysis.

455 A few limitations and assumptions of phylogenetic targeting should be noted. Although 456 the maximal pairing selects the set of species pairs that have the highest overall score 
according to a user-defined scoring model, it may select species that are not directly comparable in relation to a particular test, such as an experiment that involves testing cognitive abilities. To overcome this possible weakness, our PhyloTargeting program provides a way for the user to select pairs in which particular comparisons are possible and to

461 exclude other comparisons. Phylogenetic targeting must be used with caution if non-linear

462 relationships between the variables can be assumed, and we advise users to critically examine 463 the variables beforehand. Another critical issue is the phylogenetic tree, the representation of 464 polytomies (see Online Appendix B), and the branch lengths on which the species selection is 465 based. The selection of species can vary substantially between similar tree topologies due to 466 the fact that the maximal pairing algorithm strictly maximizes the overall score, which can 467 sometimes be heavily influenced by the topology. Branch lengths are assumed to be 468 proportional to the expected variance in the amount of evolutionary changes along each 469 branch (Brownian motion), which becomes an important assumption both in phylogenetic 470 targeting and in subsequent analyses. This is particularly true for PIC. If these assumptions are 471 violated, Type 1 error rate are inflated and statistical power is reduced (Diaz-Uriarte and 472 Garland 1996; Quader et al. 2004) . Indeed, the simulations confirmed this effect; for almost 473 all of the alternative models, Type 1 error rates were highly elevated. The only exception is 474 the early burst model, which yielded results very similar to those for Brownian motion 475 (Online Appendix C).

476 Because sister taxa will tend to be similar in many ways, confounding variables are 477 expected to be less of a problem in sister-species comparisons (Harvey and Pagel 1991; 478 Møller and Birkhead 1992). In our approach, however, more distantly related species pairs 479 can also be selected. That can be critical, because other, unmeasured confounding variables 480 may be introduced to the analysis. The comparison of distantly related species is comparable 481 to an experiment with multiple uncontrolled variables (Garland 2001; Garland and Adolph 482 1994). The more distantly related two species are, the more likely it is that such an effect 
could bias the results. By including additional variables in the calculations, it is possible to

484 control for some confounds when measurements are available.

We recommend that users standardize pairs to meet statistical requirements of

486 subsequent statistical tests (i.e., equal variances among pairs). Standardization has not

487 typically been implemented for pairwise comparisons, but it is necessary if one wishes to use

488 parametric statistical tests that make assumptions about homoskedasticity. When contrasts are

489 standardized, distantly related pairs are less often selected. This may be useful if large

490 differences are only informative when the species are closely related (e.g., to control for

491 possibly unknown confounding variables), or when comparisons should be made between

492 closely related species (e.g., because of biological differences that limit comparability of

493 experimental results). Standardization thus affects the selection of pairs.

494 Another argument for standardization is that fewer traits should change on shorter

495 branches, and thus it helps control for confounding variables. However, standardization may

496 exaggerate evolutionary differences for close relatives when differences are due to sampling

497 error or within-species variation (Purvis and Webster 1999). It can thus overestimate the

498 importance of certain species pairs if they are close relatives. We may sometimes expect a

499 larger absolute change in some trait, regardless of its rate of change, to be more valuable in

500 testing a hypothesis than a small change over a short branch. For example, brain size that

501 increases by an order of magnitude might be a stronger test than a smaller amount of brain

502 change, even if it occurs over a small branch. Using the program that we provide, the choice

503 of standardization is left up to the user (with the default option to standardize scores), based

504 on his or her preferences, the assumptions of subsequent methods, and particulars of the 505 biological system.

506 Phylogenetic targeting works best for continuous traits, but it can also be used with

507 discrete traits. However, phylogenetic targeting purely based on discrete characters is more 508 challenging because the number of distinct differences is typically smaller. In such cases, it is 
common to find that numerous pairs have the maximal possible score. This will ultimately

510 result in multiple optimal solutions in the maximal pairing algorithm. However, as the current

511 implementation returns only one optimal solution, it is difficult to evaluate its uniqueness.

512 Possible workarounds would be to either add a continuous variable or to standardize contrasts,

513 both of which help to generate variation in the scores and thus to decide among the possible 514 pairs of taxa.

515 The maximal pairing algorithm falls in a class of general combinatorial optimization 516 problems that are of considerable interest in comparative phylogenetics and bioinformatics 517 more generally. Several modifications of this algorithm have practical importance as well. 518 For example, the algorithm could be modified to select only a fixed number of pairs (given by 519 the researcher), thus incorporating the fact that limited resources are available to select species 520 for future study. This important variant has already been implemented elsewhere (see Arnold 521 and Stadler 2010). It might also be desirable to take into account conservation status of 522 different species, to ensure that species are studied before they go extinct. More generally, the 523 selection of species could be based not solely on pairwise comparisons, but on the full set of 524 contrasts, possibly in combination with examining the raw data space or regularly sampling 525 character values along the entire range of a character of interest. Here, we laid down the 526 foundations for systematically identifying species for future study. Many possible extensions 527 and modifications of the approach are possible, particularly related to alternative ways of 528 sampling species.

529 In summary, we provided a systematic method to select species for future study that 530 offers greater statistical power to test adaptive hypotheses as compared to a random selection 531 of species. With this method of phylogenetic targeting, it is also possible to control for 532 confounding variables, to incorporate alternative hypotheses, and to make use of existing data 533 on the trait of interest. It thus provides a way to guide the selection of species relative to $a$ 
534 priori hypotheses. Through our web-based computer program, other researchers are able to 535 easily implement the approach in a flexible and user-friendly way.

536

\section{Acknowledgements}

538 We want to thank all people who contributed to this research, especially Peter F. Stadler, 539 Liam Revell, and Luke J. Matthews. This research was supported by grant number BCS5400923791 from the National Science Foundation, the Max Planck Society, University of 541 Leipzig and Harvard University. 
The Maximal Pairing Problem (MPP) is the prototype of a class of combinatorial

optimization problems with considerable interest in bioinformatics and comparative phylogenetics: Given an arbitrary phylogenetic tree $\mathrm{T}$ and weights $\omega_{\mathrm{xy}}$ for the paths between any two pairs of species $(\mathrm{x}, \mathrm{y})$ (which measures the benefit or our amount of information contributed by including the comparison of species $\mathrm{x}$ with species $\mathrm{y}$ ), what is the collection of phylogenetically independent paths between pairs of leaves (i.e., no edge is shared twice) that maximizes the total weight?

In what follows, we provide algorithmic details for the implemented version for how to compute the solution of the MPP, which we call maximal pairing (MP) (see also Arnold 2008; Arnold and Stadler 2010).

The algorithm proceeds from the root of the tree up to the leaves. Solutions of subproblems (i.e., the MP of trees rooted at nodes other than the root node) are tabulated and thus do not have to be recalculated. The score for the MP for a particular tree rooted at $u$, denoted $S_{T(u)}$, can be decomposed into two cases. First, the MP of $T(u)$ may exclusively consist of pairs that do not go through $u$ itself. All pairs that contribute to $S_{T(u)}$ are thus located in the trees rooted at the children of $u$, denoted $c h d(u) . S_{T(u)}$ therefore equals the sum of $\mathrm{S}_{k}$ for each $k \in$ $\operatorname{chd}(u)$. To calculate $S_{T(u)}$, it is thus sufficient to recursively call all children of $u$.

The second case is more complex. Here, at least one pair, denoted $r_{u}$, with $u$ as the least common ancestor belongs to the MP of T(u), and $S_{T(u)}$ is thus composed of the score of $S_{r u}$ and the sum of the scores from the MP of all leftover subtrees that arise when the branches

564 from $r_{u}$ are allocated in the tree, denoted subtrees $\left(r_{u}\right)$. To calculate $S_{T(u)}$, however, we have to 565 find the particular pair $r_{u}$ that maximizes $S_{T(u)}$ for the second case (see also Figure A1). All 566 subtrees $k$ with $k \in \operatorname{subtrees}\left(r_{u}\right)$ are then called recursively. The procedure becomes much 567 more complex if polytomous nodes (degree $>2$ ) are involved, due to the fact that more than 
one pair can go through the polytomous node without violating phylogenetic independence. In

569 the current implementation, the MP algorithm calls polytomous nodes multiple times to find

570 the combination of pairs that maximizes the score of the MP for the second case by using a

571 brute force approach (for more details, see Arnold 2008).

572 These two distinct cases allow a decomposition of the initial problem into smaller 573 problems (dynamic programming). The recursions stop for subtrees with degree $=0$, i.e. the

574 tips of the tree, as their score is always 0. Ultimately, this leads to the following recursion 575 formula:

$576 S_{u}=\max \left\{\begin{array}{l}\sum_{k \in \operatorname{chd}(u)} S_{k} \\ \max _{r_{u}}\left(S_{r_{u}}+\sum_{k \in \text { subtrees }\left(r_{u}\right)} S_{k}\right)\end{array}\right.$

577 , with the notation introduced above. Figure A1 shows a graphical representation of the 578 recursion formula. After comparing the scores for both cases, the higher-scoring case is 579 selected, and the score and some additional information needed for the backtracing are 580 tabulated.

581 Finally, a backtracing procedure is applied to reconstruct the solution (i.e. the set of 582 phylogenetically independent pairs), based on the information collected in the forward 583 recursions.

584 For binary trees, the forward recursions can be computed in $O\left(n^{3}\right)$ time and $O\left(n^{2}\right)$ 585 space. If the tree is balanced, only $O\left(n^{2} \log _{2} n\right)$ time is needed. Backtracing can be computed 586 in $O\left(n^{2}\right)$ time. For polytomous nodes $p$, execution time for the MP of the tree rooted at $p$ is 587 increased exponentially by a factor $2^{\mathrm{d}-2}$ that accounts for multiple calls of $p$ (see above). 588 Execution time for polytomous trees can be improved to an overall polynomial-time algorithm 589 by building auxiliary graphs for each polytomous node and solving maximum weighted 590 matching problems (Arnold and Stadler 2010) 
592 soft polytomies are treated differently, as follows. If the polytomy is defined as hard (i.e. split 593 into more than two lineages), multiple pairs can go through the polytomous node without 594 violating phylogenetic independence. Polytomies that are defined as a series of zero-branches 595 (soft polytomies), however, are treated as a series of true dichotomies. Here, in most cases, 596 fewer pairs can be selected, due to the fact that no branch can be shared twice. Treating 597 polytomies as soft reduces execution time. Zero-length branches should be treated with 598 caution, however, since the arbitrary order of zero-branches might change the MP 599 considerably. 
602 We tested the narrow sense validity, in which the characters evolved on the randomly 603 generated tree under Brownian motion, and then investigated the broad sense validity in 604 which the characters evolved under different evolutionary models that were assumed to be 605 unknown to the user. To implement different evolutionary models, we transformed the tree 606 using the Geiger package (Harmon et al. 2008), evolved the characters with a particular model 607 on the transformed tree under Brownian motion, and used the original tree for the subsequent 608 steps. We investigated four different models that characterize stabilizing selection (the 609 Ornstein-Uhlenbeck model) (Hansen 1997), an adaptive radiation model in which most 610 change occurs early in the evolutionary history of the clade (Freckleton et al. 2003; Price 611 1997), a speciational model in which branches were equal, and a transformation of the tree 612 corresponding to weaker levels of phylogenetic signal (Freckleton et al. 2002; Pagel 1999).

613 Table B1 provides more details on the models and their parameters. 
615

All simulation results (including the results not highlighted in the manuscript) are provided in the file "Simulation results.xls".

618 
619 Ackerly, D. D. 2000. Taxon sampling, correlated evolution, and independent contrasts. Evolution 54:1480-1492.

Arnold, C. 2008. Phylogenetic Targeting: A Systematic Approach and Computer Program for Targeting Research Effort in Comparative Evolutionary Biology, University of

Arnold, C., and P. F. Stadler. 2010. Polynomial algorithms for the Maximal Pairing Problem: Efficient phylogenetic targeting on arbitrary trees. Algorithms for Molecular Biology $5: 25$

Capellini, I., P. McNamara, B. T. Preston, C. L. Nunn, and R. A. Barton. 2009. Does Sleep Play a Role in Memory Consolidation? A Comparative Test. PLoS ONE 4:e4609.

629 Clutton-Brock, T. H., and P. H. Harvey. 1977. Primate ecology and social organization. Journal of Zoology 183:1-39.

631 Cooper, N., J. Rodriguez, and A. Purvis. 2008. A common tendency for phylogenetic overdispersion in mammalian assemblages. Proceedings of the Royal Society B-

634 Diaz-Uriarte, R., and T. Garland. 1996. Testing hypotheses of correlated evolution using phylogenetically independent contrasts: sensitivity to deviations from Brownian motion. Systematic Biology 45:27-47.

637 Faustino, C. E. S. 2008. Designing a shipboard line transect survey to estimate cetacean abundance off the Azores Archipelago,Portugal, St. Andrews University, St Andrews,

640 Felsenstein, J. 1985. Phylogenies and the comparative method. American Naturalist 125:1-15.

641 Fisher, D. O., and I. P. F. Owens. 2004. The comparative method in conservation biology. Trends in Ecology \& Evolution 19:391-398. 
643 Freckleton, R. P., P. H. Harvey, and M. Pagel. 2002. Phylogenetic analysis and comparative data: A test and review of evidence. American Naturalist 160:712-726.

645 Freckleton, R. P., M. Pagel, and P. H. Harvey. 2003. Comparative methods for adaptive radiations, Pages 391-407 in T. M. Blackburn, and K. J. Gaston, eds. Macroecology: Concepts and consequences. Oxford, Blackwell.

648 Garland, T. 2001. Phylogenetic comparison and artificial selection - Two approaches in evolutionary physiology, Pages 107-132 in R. C. Roach, P. D. Wagner, and P. H.

652 Garland, T., A. F. Bennett, and E. L. Rezende. 2005. Phylogenetic approaches in comparative physiology. Journal of Experimental Biology 208:3015-3035.

654 Garland, T., A. W. Dickerman, C. M. Janis, and J. A. Jones. 1993. Phylogenetic analysis of 655 covariance by computer simulation. Systematic Biology 42:265-292.

Garland, T., P. H. Harvey, and A. R. Ives. 1992. Procedures for the analysis of comparative data using phylogenetically independent contrasts. Systematic Biology 4:18-32.

Garland, T., Jr., and S. C. Adolph. 1994. Why not to do two-species comparative studies: limitations on inferring adaptation. Physiological Zoology 67:797-828.

660 Hamilton, W. D., and M. Zuk. 1982. Heritable true fitness and bright birds: a role for parasites? Science 218:384-387.

662 Hansen, T. F. 1997. Stabilizing selection and the comparative analysis of adaptation. Evolution 51:1341-1351.

664 Harcourt, A. H., P. H. Harvey, S. G. Larson, and R. V. Short. 1981. Testis weight, body 665 weight and breeding system in primates. Nature 293:55-57. investigating evolutionary radiations. Bioinformatics 24:129-131. 
Harvey, P. H., and M. D. Pagel. 1991, The Comparative Method in Evolutionary Biology: Oxford Series in Ecology and Evolution. Oxford, Oxford University Press.

Hearn, D., and M. Huber. 2006. The ancestral distance test: What relatedness can reveal about correlated evolution in large lineages with missing character data and incomplete phylogenies. Systematic Biology 55:803-817.

673 Hosken, D. J. 1997. Sperm Competition in Bats. Proceedings of the Royal Society of London 674 - Series B: Biological Sciences 264:385-392.

Hugot, J. P. 1999. Primates and their pinworm parasites: The Cameron hypothesis revisited. Systematic Biology 48:523-546.

Lutzoni, F., M. Pagel, and V. Reeb. 2001. Major fungal lineages are derived from lichen symbiotic ancestors. Nature 411:937-940.

Lyamin, O. I., P. R. Manger, S. H. Ridgway, L. M. Mukhametov, and J. M. Siegel. 2008. Cetacean sleep: An unusual form of mammalian sleep. Neuroscience and Biobehavioral Reviews 32:1451-1484.

Maddison, D. R., D. L. Swofford, and W. P. Maddison. 1997. Nexus: An extensible file format for systematic information. Systematic Biology 46:590-621.

Maddison, W. P. 2000. Testing character correlation using pairwise comparisons on a phylogeny. Journal of Theoretical Biology 202:195-204.

Martin, R. D. 1990, Primate Origins and Evolution. London, Chapman and Hall.

Martins, E. P. 2000. Adaptation and the comparative method. Trends in Ecology and Evolution 15:296-299. $1.08(2)$.

Mitani, J. C., J. GrosLouis, and J. H. Manson. 1996. Number of males in primate groups: 
694 Moller, A. P. 1991. Sperm competition, sperm depletion, paternal care, and relative testis size in birds. American Naturalist 137:882-906.

696 Møller, A. P., and T. R. Birkhead. 1992. A pairwise comparative method as illustrated by copulation frequency in birds. American Naturalist 139:644-656.

698 Nunn, C. L., P. McNamara, I. Capellini, P. Preston, and R. A. Barton. 2009. Primate sleep in phylogenetic perspective in P. McNamara, R. A. Barton, and C. L. Nunn, eds. Evolution of sleep: Phylogenetic and Functional Perspectives. Cambridge, Cambridge University Press.

702 Nunn, C. L., and C. P. van Schaik. 2002. Reconstructing the behavioral ecology of extinct primates, Pages 159-216 in J. M. Plavcan, R. F. Kay, W. L. Jungers, and C. P. v. Schaik, eds. Reconstructing Behavior in the Fossil Record. New York, Kluwer Academic/ Plenum.

Oakes, E. J. 1992. Lekking and the evolution of sexual dimorphism in birds: comparative approaches. American Naturalist 140:665-684.

708 Pagel, M. 1999. Inferring the historical patterns of biological evolution. Nature 401:877-884.

709 Paradis, E., J. Claude, and K. Strimmer. 2004. APE: Analyses of Phylogenetics and Evolution in R language. Bioinformatics 20:289-290.

711 Price, T. 1997. Correlated evolution and independent contrasts. Philosophical Transactions of the Royal Society, London 352:519-529.

713 Purvis, A., P. M. Agapow, J. L. Gittleman, and G. M. Mace. 2000a. Nonrandom extinction and the loss of evolutionary history. Science 288:328-330.

715 Purvis, A., and L. Bromham. 1997. Estimating the transition/transversion ratio from independent pairwise comparisons with an assumed phylogeny. Journal of Molecular Evolution 44:112-119. 
718 Purvis, A., J. L. Gittleman, G. Cowlishaw, and G. M. Mace. 2000b. Predicting extinction risk in declining species. Proceedings of the Royal Society of London Series B-Biological Sciences 267:1947-1952.

721 Purvis, A., and A. Rambaut. 1995. Comparative analysis by independent contrasts (CAIC): an Apple Macintosh application for analysing comparative data. Computer Applications in the Biosciences 11:247-251.

724 Quader, S., K. Isvaran, R. E. Hale, B. G. Miner, and N. E. Seavy. 2004. Nonlinear relationships and phylogenetically independent contrasts. Journal of Evolutionary Biology 17:709-715.

727 R Development Core Team. 2009.R: A language and environment for statistical computing. R Foundation for Statistical Computing, Vienna, Austria. URL http://www.Rproject.org.

Read, A. F., and S. Nee. 1995. Inference from binary comparative data. Journal of Theoretical Biology 173:99-108.

732 Ridley, M. 1983, The explanation of organic diversity: the comparative method and adaptations of mating. Oxford, Clarendon.

734 Roth, T. C., J. A. Lesku, C. J. Amlaner, and S. L. Lima. 2006. A phylogenetic analysis of the correlates of sleep in birds. Journal of Sleep Research 15:395-402.

736 Slowinski, J. B. 1993. "Unordered" Versus "Ordered" Characters. Systematic Biology:155$737 \quad 165$

738 Webb, C. O., D. D. Ackerly, M. A. McPeek, and M. J. Donoghue. 2002. Phylogenies and community ecology. Annual Review of Ecology and Systematics 33:475-505.

740 Westoby, M. 1999. Generalization in functional plant ecology: the species sampling problem, plant ecology strategy schemes, and phylogeny, Pages 847-872 in F. I. Pugnaire, and F. Valladares, eds. Handbook of functional plant ecology. New York, M. Dekker.

743 - 2002. Choosing species to study. Trends in Ecology \& Evolution 17:587-587. 
744 Westoby, M., S. A. Cunningham, C. Fonseca, J. Overton, and I. J. Wright. 1998. Phylogeny and variation in light capture area deployed per unit investment in leaves: designs for selecting study species with a view to generalizing, Pages 539-566 in H. Lambers, H. Poorter, and M. M. I. V. Vuren, eds. Variation in growth rate and productivity of higher plants. Leiden, The Netherlands, Backhuys Publishers. 


\section{FIGURES}

752 Figure 1. Flow chart for applying phylogenetic targeting. Phylogenetic targeting is essentially

753 a taxon sampling technique to systematically guide future data collection.

755 Figure 2. Three out of the 15 possible pairings for an example tree. Paired species are highlighted in black. One pairing has three pairs, ten pairings two pairs, and four only one pair. In all pairings, pairs are phylogenetically independent, and no additional pair can be added without violating the requirement of phylogenetic independence.

Figure 3. Example dataset and phylogeny for applying phylogenetic targeting. The tree shows continuously varying traits $X_{1}, X_{2}, Y_{t}$ and a binary trait $B_{t}$ indicating whether the species has already been studied in relation to $Y_{t}$. Two species have already been studied regarding $Y_{t}$, and data on $Y_{t}$ are missing for four species. The goal is to identify which of the four unstudied species should be targeted for studying $Y_{t}$.

Figure 4. Results from the simulations. Simulation results for the percentage of the used range of variation for $X_{l}$ when species pairs are selected using phylogenetic targeting (dark grey) and randomly (light grey) are shown. The x-axis plots the effects of the number of pairs that have been selected $(6,9$, and 12$)$. Contrast standardization is turned on.

Figure 5. Selected results from the simulations under Brownian motion. Type I errors and

772 statistical power for correlation tests based on pairwise comparisons (PC, left category) and 773 phylogenetically independent contrasts (PIC, right category) are shown for phylogenetically 774 targeted sampling ("PT") and random taxon sampling ("R"). The first three bars in each 775 category represent Type I error rates (based on 50, 70, and 90 species tree; from left to right), 
776 and the last three bars represent statistical power (also based on 50, 70, and 90 species tree;

777 from left to right). Contrast standardization is turned on, and six pairs were selected.

778

779 Figure A1. Graphical representation of the recursion formula of the maximal pairing

780 algorithm for bifurcating nodes. Calculation of the maximal pairing proceeds recursively from

781 the root to the tips. For each internal node, two distinct cases can be distinguished that allow a

782 decomposition of the initial problem into smaller problems (dynamic programming). The

783 higher-scoring case is selected and the recursion proceeds. Note that for polytomous nodes, a

784 different algorithm is used (not shown here). See text for details.

785

p. 35 
TABLE 1. ILLUSTRATION OF THE SCORING SYSTEM AND THE MAXIMAL PAIRING, APPLIED TO FIGURE 2.

\begin{tabular}{|c|c|c|c|c|c|c|c|c|c|}
\hline \multirow{3}{*}{$\begin{array}{c}\text { Pairwise } \\
\text { comparison }\end{array}$} & \multicolumn{2}{|c|}{$X_{1}$} & \multicolumn{4}{|c|}{$X_{2}$} & \multirow{3}{*}{$\begin{array}{c}\text { Summed } \\
\text { score }\end{array}$} & \multirow{3}{*}{$\begin{array}{c}\text { Sum of branch } \\
\text { lengths }\end{array}$} & \multirow{3}{*}{$\begin{array}{l}\text { Standardized } \\
\text { summed score }\end{array}$} \\
\hline & \multirow{2}{*}{$\Delta_{\text {Raw }}$} & \multirow{2}{*}{ Score } & \multirow{2}{*}{$\Delta_{\text {Raw }}$} & \multicolumn{3}{|c|}{ Score } & & & \\
\hline & & & & Score $_{\mathrm{NC}}$ & Score $_{\text {SD }}$ & Score $_{\mathrm{OD}}$ & & & \\
\hline $\mathrm{s} 1-\mathrm{s} 2 *$ & 0.5 & 0.385 & -3 & 0.831 & -0.171 & 0.171 & 1.216 & 6 & 0.496 \\
\hline s1-s3 & 0.8 & 0.615 & -1.5 & 0.916 & -0.086 & 0.086 & 1.531 & 6 & 0.625 \\
\hline s1-s4 & 1.3 & 1 & -2.7 & 0.848 & -0.154 & 0.154 & 1.848 & 6 & 0.755 \\
\hline s1-s5 & 1 & 0.769 & 14.8 & 0.169 & 0.831 & -0.831 & 0.938 & 8 & 0.332 \\
\hline s1-s6 & 0.6 & 0.462 & 9.6 & 0.461 & 0.539 & -0.539 & 0.922 & 8 & 0.326 \\
\hline s2-s3 & 0.3 & 0.231 & 1.5 & 0.916 & 0.084 & -0.084 & 1.146 & 4 & 0.573 \\
\hline $\mathrm{s} 2-\mathrm{s} 4$ & 0.8 & 0.615 & 0.3 & 0.983 & 0.017 & -0.017 & 1.599 & 4 & 0.799 \\
\hline s2-s5 & 0.5 & 0.385 & 17.8 & 0 & 1 & -1 & 0.385 & 8 & 0.136 \\
\hline s2-s6 & 0.1 & 0.077 & 12.6 & 0.292 & 0.708 & -0.708 & 0.369 & 8 & 0.13 \\
\hline s3-s4* & 0.5 & 0.385 & -1.2 & 0.933 & -0.069 & 0.069 & 1.317 & 2 & 0.931 \\
\hline
\end{tabular}




$\begin{array}{lccccccccc}\text { s3-s5 } & 0.2 & 0.154 & 16.3 & 0.084 & 0.916 & -0.916 & 0.238 & 8 & 0.084 \\ \text { s3-s6 } & 0.2 & 0.154 & -11.1 & 0.376 & -0.634 & 0.634 & 0.53 & 8 & 0.187 \\ \text { s4-s5 } & 0.3 & 0.231 & -17.5 & 0.017 & -1 & 1 & 0.248 & 8 & 0.088 \\ \text { s4-s6 } & 0.7 & 0.538 & -12.3 & 0.309 & -0.703 & 0.703 & 0.847 & 8 & 0.3 \\ \text { s5-s6* } & 0.4 & 0.308 & 5.2 & 0.708 & 0.292 & -0.292 & 1.016 & 2 & 0.718\end{array}$

NOTE. $-\Delta_{\text {Raw }}=$ raw difference of trait values (see Figure 2). See scoring section for details on Score $\mathrm{NC}_{\text {, }}$ Score $\mathrm{SD}_{\mathrm{S}}$, and 


\begin{tabular}{|c|c|c|}
\hline Model of evolution & Description of the model & Parameters in the GEIGER package \\
\hline Brownian motion & constant-rate random-walk model & None \\
\hline Ornstein-Uhlenbeck & $\begin{array}{l}\text { random-walk model with a central tendency, so } \\
\text { that phenotypes tend to evolve towards one } \\
\text { "optimal" value }\end{array}$ & $\alpha=0.5,1$, and 2 \\
\hline Adaptive radiation / Early burst & $\begin{array}{l}\text { rate of evolution decays exponentially through } \\
\text { time }\end{array}$ & endRate $=0.3$ and 0.6 \\
\hline Speciational/ Punctuated & all branches have length 1 & None \\
\hline Lambda transformation & $\begin{array}{l}\text { The parameter } \lambda \text { is a scaling parameter that can } \\
\text { be used to estimate phylogenetic signal. } \\
\text { Decreasing the value of } \lambda \text { has the effect of } \\
\text { gradually eliminating phylogenetic structure. } \\
\text { Under Brownian motion, } \lambda \text { takes the value } 1.0 \\
\text { by default. If the Brownian motion assumption } \\
\text { is violated, however, } \lambda \text { will significantly depart } \\
\text { from } 1.0 \text {. }\end{array}$ & $\lambda=0.3$ and 0.6 \\
\hline
\end{tabular}




\section{Fig. 1}

\section{Problem:}

Given resource limitations in collecting data and a specific hypothesis, which species should be studied to increase the size of comparative datasets?

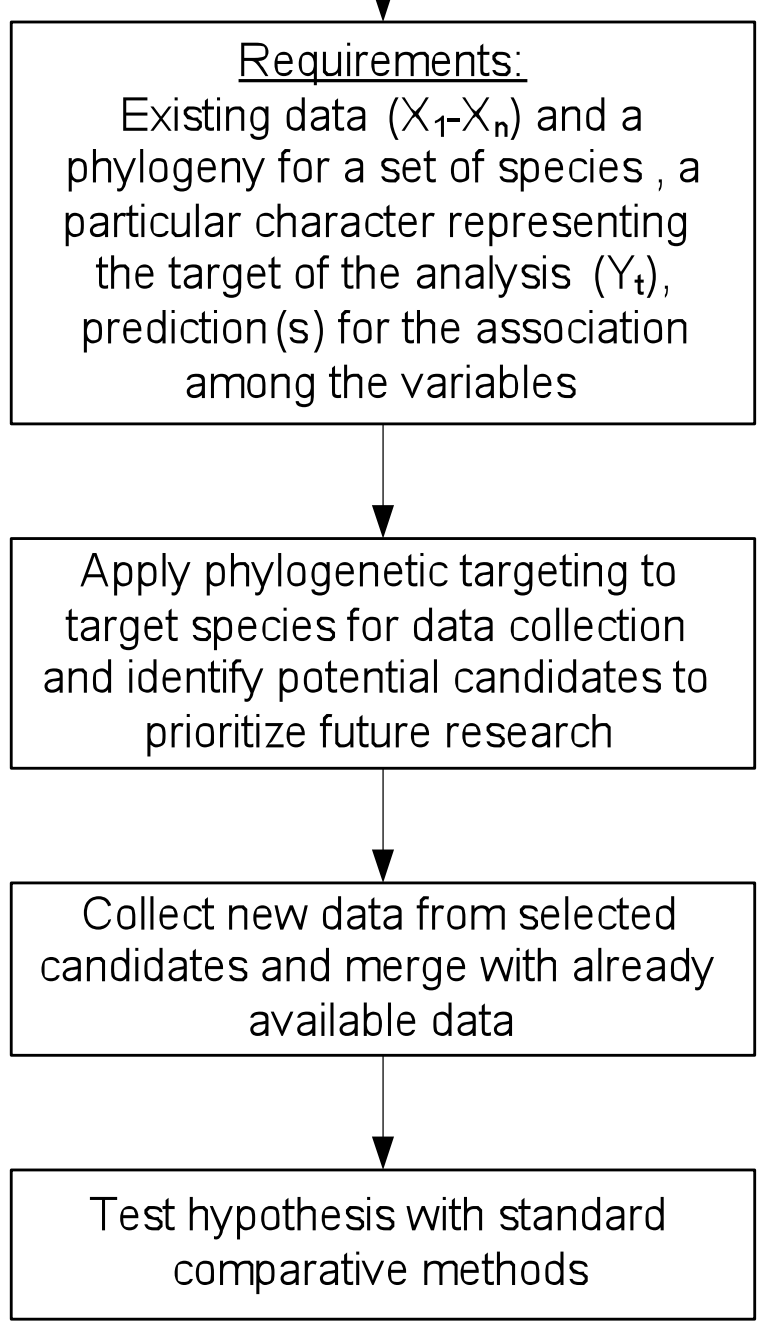


Fig. 2
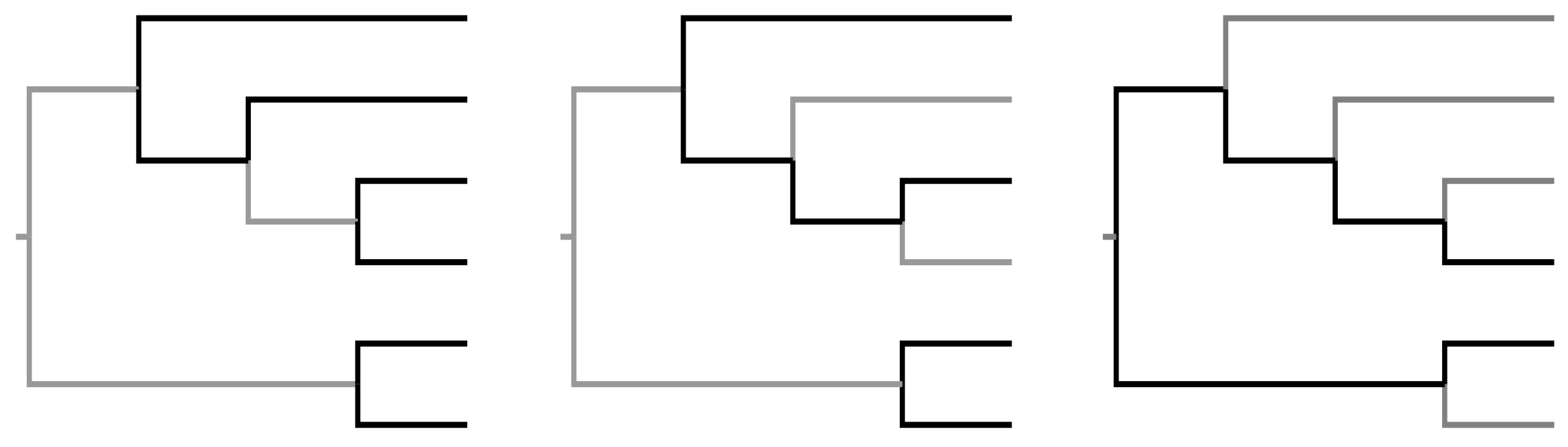
Fig. 3

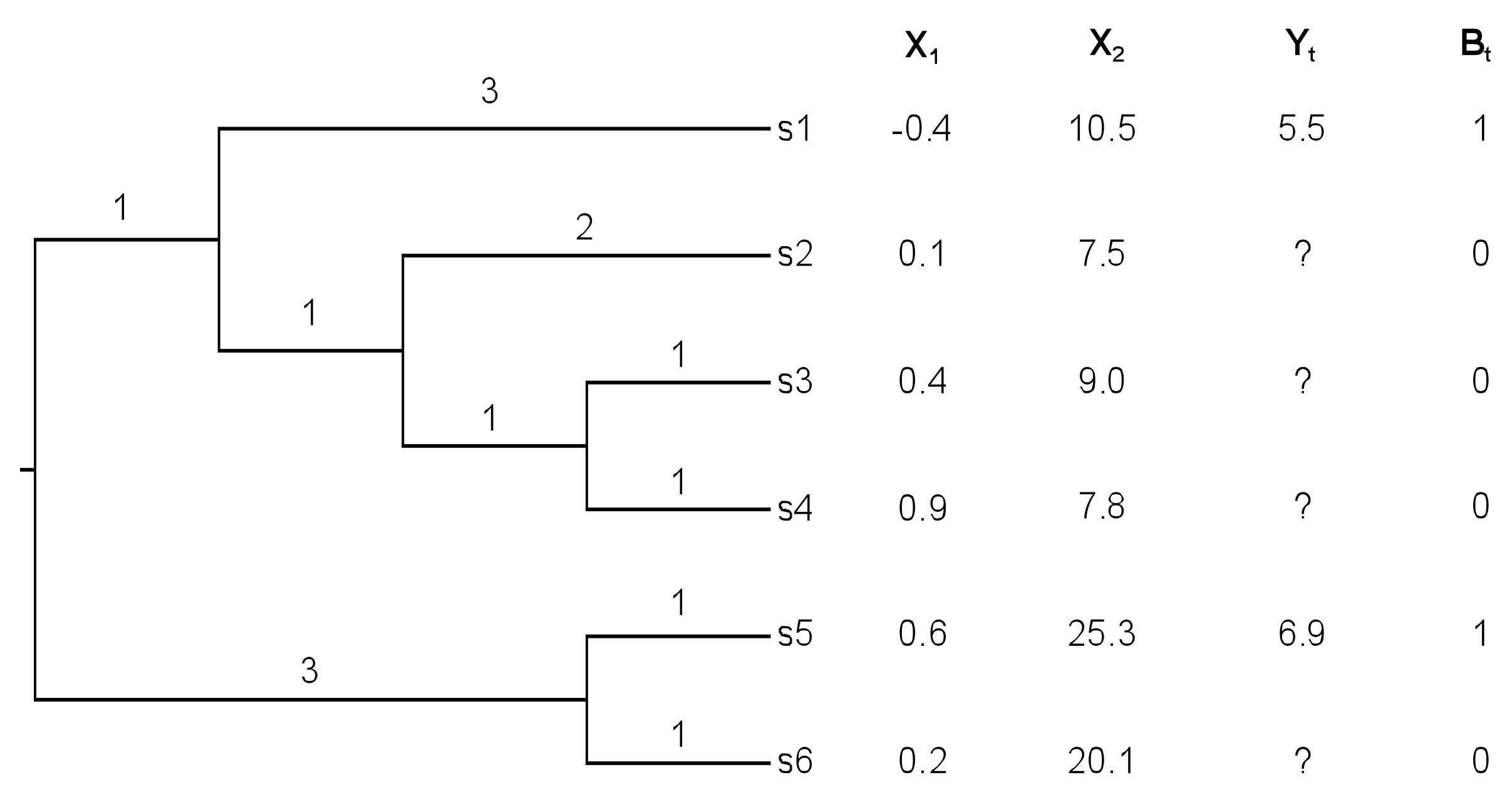




$$
\text { III }
$$




\section{Fig. 5}

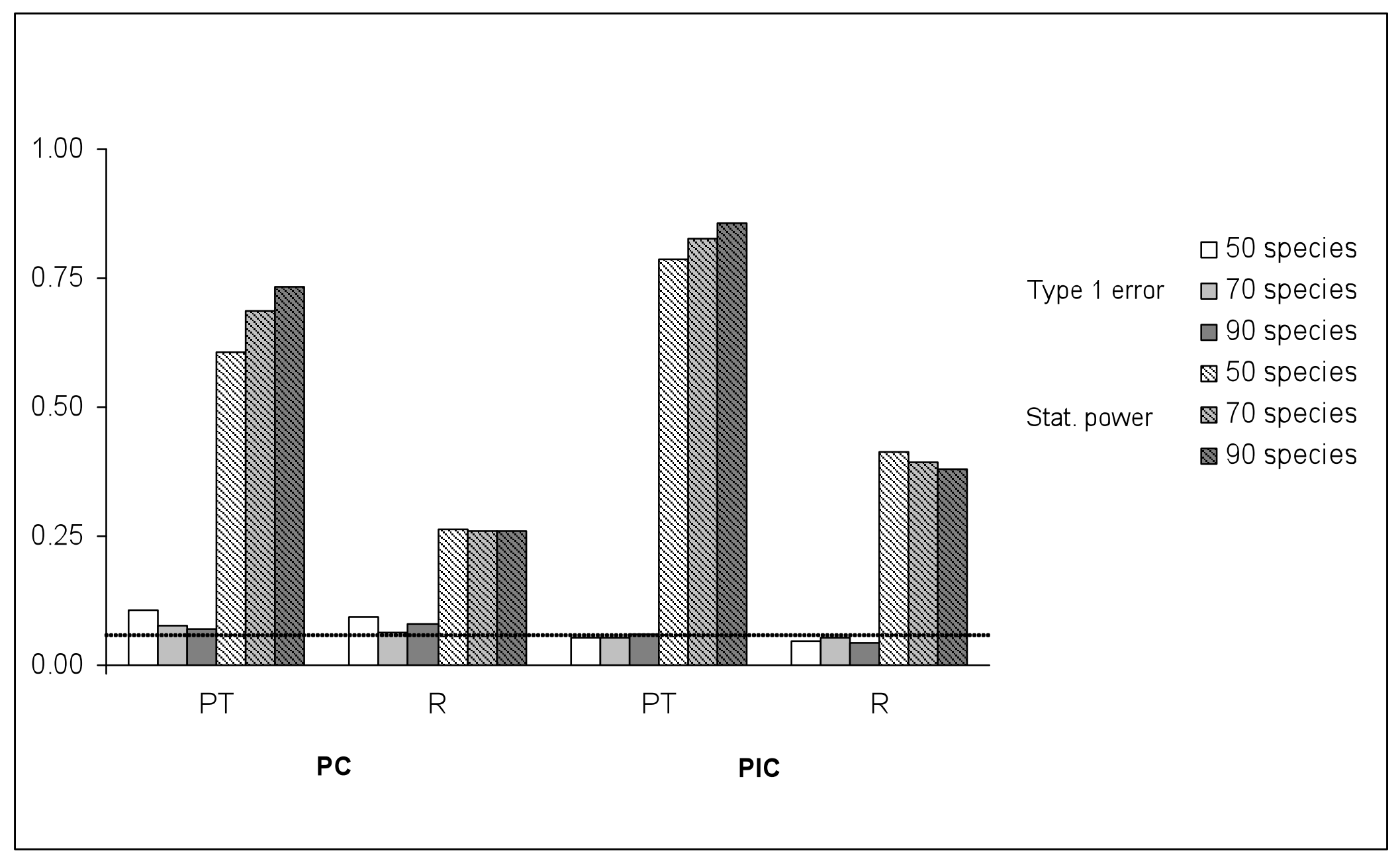


Fig. A1

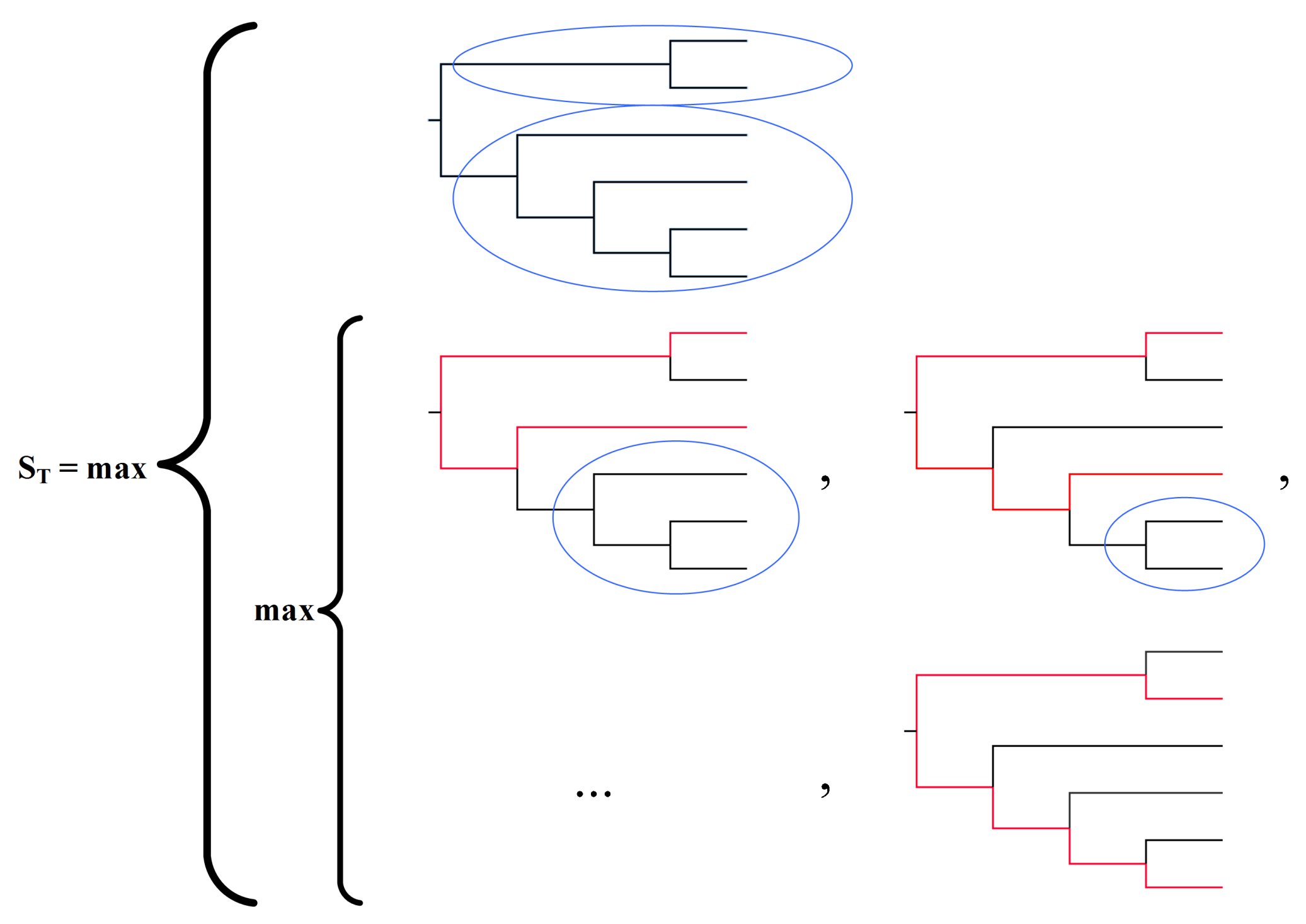

